\title{
Verso il Centenario dello scoppio della Grande guerra. Intervista a Patrizia Dogliani e Giovanna Procacci
}

ISSN: 2282-4979

DOI: 10.12977/ereview58 Questo articolo è stato sottoposto a un processo di double blind peer review

L'obiettivo di questa doppia intervista a Patrizia Dogliani e Giovanna Procacci è offrire uno sguardo critico sullo stato attuale della storiografia sulla Grande guerra. L'attenzione è posta in particolare sui temi che appaiono più significativi nel centenario dell'evento, come la memoria pubblica, le celebrazioni, i nuovi percorsi di ricerca, in una prospettiva comparativa che intende mettere a fuoco la situazione italiana collocandola nello scenario europeo.

The purpose of this double interview with Patrizia Dogliani and Giovanna Procacci is to offer a critical view about the current state of the historiography on the Great War. The focus is particularly on the more significant issues in the centenary of the historical event, such as the public memory, the celebrations, the new research approaches, in a comparative perspective that observes the Italian case in the European background.

(Contributo video disponibili online: 目国 http://youtu.be/620pIrXUx2I).

A poche settimane dal centenario dello scoppio della Grande guerra, si è ritenuto opportuno introdurre questo dossier, dedicato al tema La Grande guerra in retrovia, facendo ricorso ad alcune riflessioni emerse da due videointerviste che Patrizia Dogliani e Giovanna Procacci ci hanno voluto gentilmente concedere con l'intento di fare il punto su alcune questioni cruciali, quali la memoria pubblica della Grande guerra e i percorsi storiografici connessi a questo evento.

Da alcuni mesi un numero crescente di addetti ai lavori e semplici osservatori si dichiarano colpiti dal grado di sostanziale immobilismo con cui lo stato italiano si sta apprestando a celebrare questo importante anniversario della storia nazionale ed europea. L'apposito comitato storico scientifico istituito presso la 
Presidenza del Consiglio dei Ministri nell'agosto del 2012, a cui era stato affidato il «coordinamento della pianificazione, della preparazione e dell'organizzazione degli interventi connessi alle celebrazioni», non sembra aver fin qui prodotto un programma organico e puntuale di interventi sia sul piano delle celebrazioni ufficiali, sia sul piano di una vasto impegno finalizzato a rilanciare e innovare gli studi sulla Prima guerra mondiale. In mancanza di risorse economiche adeguate e con il venir meno di una spinta politica decisa da parte degli esecutivi nazionali, che si sono succeduti nel corso degli ultimi tre anni, il comitato sembra ad oggi non essere in grado di svolgere la sua funzione di coordinamento unitario a livello nazionale. Di fatto, osserva Giovanna Procacci, l'impressione è che, mancando i fondi economici necessari, il comitato tenda semplicemente a patrocinare iniziative già finanziate.

A oggi, il centenario non sembra poter rappresentare l'occasione per l'avvio deciso di un processo di nazionalizzazione della storia e della memoria pubblica della Grande guerra. Al contrario, il vuoto di coordinamento sopra richiamato è stato colmato dai governi regionali, particolarmente delle regioni del Nord est (Veneto, Trentino Alto Adige e Friuli Venezia Giulia, queste ultime due regioni a statuto speciale in grado di mettere in campo risorse economiche importanti) che sono state attraversate dal fronte militare e al cui interno la memoria della Grande guerra è sempre stata maggiormente presente rispetto al resto del territorio nazionale. Si stanno dunque profilando i rischi di un'ulteriore regionalizzazione della memoria del primo conflitto mondiale e del venir meno della possibilità di avviare una nuova fase di ricerche di ampio respiro storiografico: «Se queste regioni - osserva Patrizia Dogliani - non si agganciano a centri di ricerca che possono promuovere ricerche scientificamente valide e di ampio respiro storiografico, il loro attivismo correrebbe il rischio di rimanere confinato ad intenti di carattere puramente celebrativo o limitato alla sola valorizzazione dei propri territori sul piano del turismo di guerra».

Questa frammentarietà d'iniziative locali, ad oggi prive di una direzione nazionale che risulti in maniera chiara nella definizione dei contenuti di questi interventi e nelle modalità di comunicazione, emerge ancora più evidente se si effettua una comparazione con quanto accade fuori dai confini nazionali, con particolare riferimento ai contesti francese, inglese e tedesco. Internet ci consegna la presenza di portali web appositamente organizzati dai singoli governi, in grado di restituirci con evidenza questa volontà di coordinamento centrale che si esplicita nella presentazione di programmi di lavoro già ben definiti e strutturati. Al contrario, per quel che riguarda il caso italiano, la rete promuove un quadro d'iniziative, in qualche caso certamente interessanti, ma assolutamente frammentarie e non in 
grado di stimolare un necessario ed auspicabile rinnovamento storiografico. Il rischio sopra richiamato - con riferimento all'immobilismo del governo nazionale cui fa da contraltare un attivismo locale di pochissime regioni, chiaramente individuabili sul piano geografico in un'area ben definita della penisola - ossia di incentivare una sorta di «storiografia a chilometro zero», è reso ancor più reale e preoccupante dal permanere di una profonda perifericità della storiografia nazionale italiana all'interno del consesso scientifico europeo. In Europa - ha sottolineato Patrizia Dogliani - ormai da alcuni anni, i maggiori centri di ricerca (Universitat freie di Berlino, Historial de la Grande Guerre di Peronne, Trinity college di Dublino) lavorano in modo puntuale e concertato attorno alla raccolta di dati, alla costruzione di reti e alla programmazione di convegni sempre più proiettati a rafforzare l'idea della Grande guerra come guerra europea e soprattutto mondiale, superando la visione di un evento bellico a lungo interpretato come una guerra sostanzialmente franco tedesca, e proiettando lo sguardo anche in direzione dei paesi ex Commonwealth (Australia, Canada, Nuova Zelanda e così via). La stessa storiografia tedesca che dopo la riunificazione aveva indagato con attenzione il periodo degli anni Trenta e Quaranta, da alcuni anni si sta dedicando con interesse crescente allo studio della Prima guerra mondiale. Un quadro arricchito anche dall'attivismo degli storici spagnoli e portoghesi, particolarmente impegnati nel tentativo di fornire alle proprie ricerche un respiro europeo, attraverso il rinnovamento degli studi su intellettuali europei e Grande guerra e la promozione di convegni riservati al tema della neutralità; e quindi al rapporto tra Spagna e Portogallo - che entrerà in guerra nel 1916 - e paesi in guerra. Sul piano della storia strettamente politica, la storiografia sulla classe operaia e sull'internazionalismo che da lungo tempo taceva, sembra riemergere offrendo l'opportunità di una rilettura più approfondita della storia europea della prima metà del secolo scorso in grado di favorire una ricostruzione più puntuale $\mathrm{e}$ articolata del passaggio che va dalla fine dell'internazionalismo all'emergere dei nazionalismi e all'affermazione di un certo transnazionalismo, che domina gli anni tra le due guerre. Rimanendo all'interno del perimetro della storia politica, è necessario osservare che la riscoperta degli studi sulla dissoluzione dei grandi imperi - che sembrerebbe emergere in quest'ultimo periodo - potrebbe agevolare dal canto suo una ricostruzione della storia del vecchio continente non più fortemente vincolata all'analisi degli assetti geopolitici definiti alla fine della Seconda guerra mondiale.

All'interno di questo contesto europeo di crescente attenzione verso la storia della Grande guerra, in cui si segnala il rafforzamento e, per molti versi, il rinnovamento - sia a livello dei temi indagati, sia a livello di un tentativo sempre più 
deciso in direzione di un approccio comparativo sovranazionale - delle grandi storiografie nazionali e il tentativo di inserimento di gruppi di ricerca quali quello spagnolo e portoghese, la storiografia italiana continua ad avere un ruolo marginale. L'impressione è che tale ritardo non sia imputabile alla sfasatura tra l'anno dello scoppio del conflitto (1914) e l'anno in cui l'Italia entrò in guerra (1915). Semmai, il sostanziale mancato inserimento della comunità degli storici italiani all'interno delle reti promosse dai maggiori centri di ricerca europei sembrerebbe poter essere ricondotto al permanere di «un sentimento di perifericità» rispetto al contesto scientifico continentale.

L'interpretazione, a lungo dominante nel panorama storiografico europeo, secondo cui la Grande guerra fu essenzialmente una guerra franco tedesca, ha fatto si che l'Italia - come osserva Giovanna Procacci - sia stata a lungo considerata «un paese di secondarissima importanza»; i militari italiani non hanno preso parte alle battaglie sui grandi fronti europei, non erano a Verdun o su La Somme; mentre il fronte italiano è sempre stato ritenuto marginale ai fini dell'esito finale della guerra.

D'altra parte, in Italia il primo conflitto mondiale del Novecento è stato descritto per molto tempo non come una grande guerra europea, ma piuttosto come l'ultima guerra risorgimentale. L'interpretazione della guerra del '15-' 18 fornita dal regime fascista prima e la critica decisa che la storiografia militante di fine anni Sessanta ha rivolto ai simboli di quell'evento, come la festa nazionale del 4 novembre considerata espressione della retorica militarista, hanno fatto si che esso venisse allontanato dalle letture popolari e anche dall'humus della tradizione democratica nazionale.

Sul piano strettamente legato alla ricerca storica, alcuni punti di eccellenza emersi nel corso dei decenni precedenti all'interno del dibattito storiografico italiano - dagli studi sul movimento operaio, all'analisi del fenomeno della diserzione, della follia o della dimensione della prigionia (con riferimento agli studi di Enzo Forcella e Alberto Monticone [1968], Bruna Bianchi [2001; 2006], Antonio Gibelli [1991], Giovanna Procacci [1999; 2000; 2013] e Patrizia Dogliani [1983] ) - che si sono rivelati anticipatori di filoni d'indagine poi ampiamente sviluppatisi in altri contesti nazionali di ricerca, non sono stati seguiti negli anni recenti da altri percorsi di ricerca altrettanto fortunati. In qualche misura, contrariamente a quanto avvenuto in Francia, dove la storiografia nazionale ha avuto una stratificazione di storici su almeno tre generazioni, che si sono succedute e confrontate attorno ai temi della Grande guerra, in Italia è mancato un passaggio del testimone tra generazioni di storici attenti alle vicende di quegli anni.

A ben guardare, inoltre, dentro l'accademia italiana la Grande guerra «gode di 
poca fortuna», e sperimenta una posizione di assoluta subalternità rispetto alla Seconda guerra mondiale e ad alcuni ambiti tematici ad essa legati, quali la persecuzione ebraica, la prigionia di guerra dei soldati italiani, la vicenda della Repubblica sociale italiana e della guerra di liberazione.

All'interno di questo contesto non particolarmente favorevole, dove sempre meno storici si dedicano allo studio di quella guerra, gli studi prodotti tendono in prevalenza a rigettare la dimensione di uno sguardo comparativo che superi i confini nazionali. Tornando alla contingenza del Centenario, sono quasi inesistenti i gruppi di ricerca italiani che stanno promuovendo momenti di confronto in grado di coinvolgere studiosi stranieri (tra le eccezioni citiamo le iniziative messe in atto dall'Istituto storico italo germanico di Trento e dal gruppo di ricerca che opera all’Università Ca' Foscari di Venezia e fa capo a Bruna Bianchi). Il «sentimento di perifericità», sopra ricordato, sembra dunque permanere.

La stessa editoria nazionale, poco incline a promuovere la pubblicazione di libri dedicati a questo evento, meno fortunati sul piano commerciale rispetto a saggi centrati sulla storia della Seconda guerra e ai temi sopra ricordati, tende a privilegiare i testi scritti da autori stranieri, prevalentemente di provenienza anglosassone, che abitualmente producono lavori di grande respiro narrativo adatti ad un grande pubblico, piuttosto che ad una ristretta cerchia di ricercatori ed esperti della materia.

Il Centenario - concordano Patrizia Dogliani e Giovanna Procacci - deve rappresentare per la comunità degli storici italiani l'occasione per fare il punto sulle ricerche condotte nel periodo successivo alla fortunata stagione di studi sopra richiamata e conclusasi nei primi anni Novanta (Giovanna Procacci ha recentemente curato per gli annali della Fondazione La Malfa un testo dedicato alla società italiana in guerra in cui si fa il punto su alcuni grandi temi) al fine di valorizzare i percorsi di studio - come quelli legati alla storia di genere, al tema della violenza sui civili e allo studio dell'apparato repressivo messo in campo dallo stato per controllare la società civile - che hanno prodotto risultati significativi e individuare nuovi percorsi di ricerca che valga la pena percorrere. Su questo aspetto, Giovanna Procacci sostiene la necessità di tornare agli studi di storia delle istituzioni, del potere dello stato, al rapporto tra potere civile e potere militare e come questi si intersecano nella Prima guerra mondiale anche nella vita civile dei singoli cittadini. Ma soprattutto occorre promuovere ricerche di storia sociale in grado fornirci informazioni sulla vita quotidiana della gente comune nelle città e nelle campagne nel corso di quegli anni.

Date queste considerazioni, il dossier dedicato ai temi delle retrovie e del fronte interno, punta a mettere in risalto le peculiarità della regione Emilia-Romagna 
negli anni della Grande guerra.

La scelta della scala regionale non è casuale, pure nella consapevolezza delle peculiarità provinciali o dei singoli comuni. La guerra è stata vissuta in modi differenti all'interno della regione a seconda della possibilità di reperimento delle risorse, delle difficoltà nei collegamenti ferroviari e stradali, dell'accessibilità al mare, della presenza di ospedali e di altre strutture sanitarie, dell'insediamento di truppe, dell'atteggiamento e delle scelte operate dalle amministrazioni locali di diverso orientamento politico. Ciò detto, ci siamo orientati a un'analisi dell'Emilia Romagna nel suo complesso, ritenendo che esistano specificità da cogliere allargando la scala di analisi.

Ad oggi mancano studi complessivi sulla regione Emilia Romagna nella Prima guerra mondiale e nemmeno il Centenario sembra rappresentare, da questo punto di vista, una svolta. Anche in questo caso spiace rilevare, a livello istituzionale e di centri di ricerca, il ritardo nella messa a punto di un progetto complessivo che possa indagare, come invece è stato fatto per tanti studi sul «modello emiliano» del secondo dopoguerra, le specificità di questo territorio. Il rischio, in questo caso, non è quello di una «regionalizzazione della memoria» - richiamato da Patrizia Dogliani per le regioni del fronte militare - ma al contrario di perpetuare l'idea (ampiamente diffusa) che la Prima guerra mondiale lontana dal fronte abbia seguito dinamiche omogenee su tutto il territorio nazionale e che pertanto non sia rilevante analizzarne gli aspetti peculiari in contesti più ristretti.

È nostra convinzione, supportata dai primi risultati dei contributi pervenuti per il dossier, che le caratteristiche del territorio, il suo grado di sviluppo, le azioni e le reazioni dei decision-makers locali abbiano invece marcato una «singolarità emiliana» anche nella Prima guerra mondiale; una singolarità che, dati i limiti di questo lavoro, può essere qui soltanto abbozzata, ma che andrebbe invece verificata ed approfondita con analisi comparate di più ampio respiro, non soltanto nazionale. Come già accennato, in questa direzione non vediamo all'orizzonte progetti strutturati in ambito regionale e nemmeno italiano, ma solo sporadici contributi. Pregevoli tentativi di comparazione a livello europeo - come il saggio scritto di recente da Felicita Ratti sull'origine, la diffusione e gli effetti dell'influenza spagnola nella provincia di Modena e nel Land Salisburgo - non circolano come dovrebbero nelle sedi accademiche e nei centri di ricerca.

Le piste di ricerca che si possono seguire in ambito regionale per marcare specificità e differenze sono tante e potrebbero fornire interessanti occasioni per superare da un lato il rischio del «regionalismo» e dall'altro quel «sentimento di perifericità» cui si accennava in precedenza.

In questo dossier indichiamo alcune tra le piste possibili, che partendo dalla spe- 
cificità emiliana, possano fornire chiavi di lettura più generali. Si pensi, ad esempio, all'impatto della prima guerra tecnologica e industriale della storia su un sistema della cura come quello emiliano-romagnolo, che alla vigilia del conflitto si stava modernizzando. In particolare nei settori dell'ortopedia, della fisioterapia e della psichiatria la guerra impresse uno sviluppo senza precedenti e lasciò tracce durevoli nel tempo. Si pensi inoltre alle avanzate politiche di gestione dei consumi che - come nel caso di Bologna con il Sindaco Francesco Zanardi e di Reggio Emilia con Luigi Roversi - vennero precocemente attivate per far fronte al rincaro dei generi di prima necessità, configurando i prodromi di un più moderno welfare locale; o, ancora, alla gestione dell'emergenza profughi, affluiti copiosi da Veneto e dal Friuli fin dal 1916 ma soprattutto nel 1917, che esercitarono un'inedita pressione sul territorio regionale, inducendo le amministrazioni locali a sperimentare e innovare.

\section{Bibliografia}

Bianchi B. 2001, La follia e la fuga. Nevrosi di guerra, diserzione e disobbedienza nell'esercito italiano, 1915-1918, Roma: Bulzoni.

Bianchi B. 2006, La violenza contro la popolazione civile nella grande guerra: deportati, profughi, internati, Milano: Unicopli.

Dogliani P. 1983, La scuola delle reclute. L'Internazionale giovanile socialista dalla fine dell'Ottocento alla Prima guerra mondiale, Torino: Einaudi

Forcella E. e Monticone A. 1968, Plotone d'esecuzione. I processi della prima guerra mondiale, Bari: Laterza.

Gibelli A. 1991, L'officina della guerra. La grande guerra e le trasformazioni del mondo mentale, Torino: Bollati Boringhieri.

Procacci G. 1999, Dalla rassegnazione alla rivolta. Mentalità e comportamenti popolari nella grande guerra, Roma: Bulzoni.

Procacci G. 2000, Soldati e prigionieri italiani nella Grande guerra, Torino: Bollati Boringhieri.

Procacci G. 2013, Warfare-welfare. Intervento dello Stato e diritti dei cittadini (1914-18), Roma: Carocci.

Ratti F. 2010, "Qui sono diventati 'spagnoli' in molti". Storia sociale comparata della pandemia influenzale 1918-1919 nella provincia di Modena e nel Land Salisburgo, in Montella, F., Paolella, F., Ratti, F., Una regione ospedale, Bologna: Clueb. 


\section{Risorse}

Documenti e immagini della Grande Guerra

http://www.14-18.it

Europeana 1914-1918

http://www.europeana1914-1918.eu/it

First World War Centenary

https://www.gov.uk/government/topical-events/first-world-war-centenary

Centenario della Grande Guerra 1914-1918

http://www.france.fr/it/istituzioni-e-valori/il-centenario-della-grande-guerra-1914-1918.html

World Imperial Museum

http://www.1914.org

Mission Centenaire

http://centenaire.org/fr 